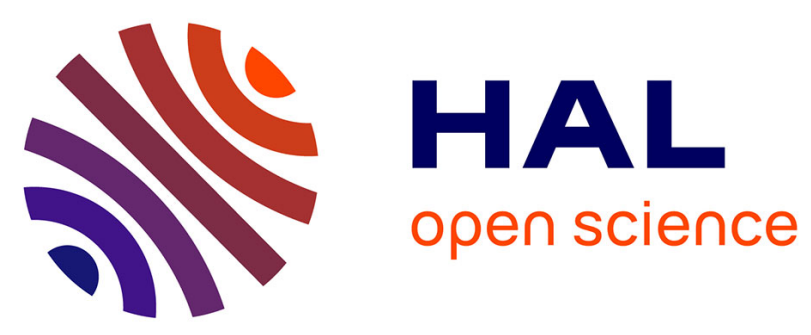

\title{
DUST FROM COMET 209P/LINEAR DURING ITS 2014 RETURN: PARENT BODY OF A NEW METEOR SHOWER, THE MAY CAMELOPARDALIDS
}

\author{
Masateru Ishiguro, Daisuke Kuroda, Hidekazu Hanayama, Jun Takahashi, \\ Sunao Hasegawa, Yuki Sarugaku, Makoto Watanabe, Masataka Imai, Shuhei \\ Goda, Hiroshi Akitaya, et al.
}

\section{- To cite this version:}

Masateru Ishiguro, Daisuke Kuroda, Hidekazu Hanayama, Jun Takahashi, Sunao Hasegawa, et al.. DUST FROM COMET 209P/LINEAR DURING ITS 2014 RETURN: PARENT BODY OF A NEW METEOR SHOWER, THE MAY CAMELOPARDALIDS. The Astrophysical journal letters, 2015, 798, pp.L34. 10.1088/2041-8205/798/2/L34 . hal-01203039

\section{HAL Id: hal-01203039 \\ https://hal.science/hal-01203039}

Submitted on 24 Sep 2015

HAL is a multi-disciplinary open access archive for the deposit and dissemination of scientific research documents, whether they are published or not. The documents may come from teaching and research institutions in France or abroad, or from public or private research centers.
L'archive ouverte pluridisciplinaire HAL, est destinée au dépôt et à la diffusion de documents scientifiques de niveau recherche, publiés ou non, émanant des établissements d'enseignement et de recherche français ou étrangers, des laboratoires publics ou privés.

\section{(2)(1) $\$$}

Distributed under a Creative Commons Attribution - NonCommercial - ShareAlikel 4.0 


\title{
DUST FROM COMET 209P/LINEAR DURING ITS 2014 RETURN: PARENT BODY OF A NEW METEOR SHOWER, THE MAY CAMELOPARDALIDS
}

\author{
Masateru Ishiguro $^{1,20}$, Daisuke Kuroda ${ }^{2}$, Hidekazu Hanayama $^{3}$, Jun Takahashi $^{4}$, Sunao Hasegawa ${ }^{5}$, Yuki $_{\text {Sarugaku }}{ }^{2}$, \\ Makoto Watanabe ${ }^{6}$, Masataka Imai $^{6}$, Shuhei Goda ${ }^{6}$, Hiroshi Akitaya ${ }^{7}$, Yuhei Takagi ${ }^{4}$, Kumiko Morihana $^{4}$,

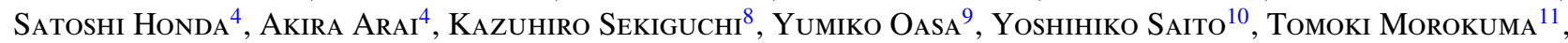 \\ Katsuhiro Murata ${ }^{12}$, Daisaku Nogami ${ }^{13}$, Takahiro Nagayama ${ }^{14}$, Kenshi Yanagisawa ${ }^{2}$, Michitoshi Yoshida ${ }^{15}$, \\ Kouji Ohta ${ }^{16}$, Nobuyuki Kawai ${ }^{10}$, Takeshi Miyaji ${ }^{3}$, Hideo Fukushima ${ }^{17}$, Jun-IChi Watanabe ${ }^{17}$, \\ Cyrielle Opitom $^{18}$, Emmanuël Jehin ${ }^{18}$, Michael Gillon ${ }^{18}$, and Jeremie J. Vaubaillon ${ }^{19}$ \\ ${ }^{1}$ Department of Physics and Astronomy, Seoul National University, Gwanak, Seoul 151-742, Korea \\ ${ }^{2}$ Okayama Astrophysical Observatory, National Astronomical Observatory of Japan, Asakuchi, Okayama 719-0232, Japan \\ ${ }^{3}$ Ishigakijima Astronomical Observatory, National Astronomical Observatory of Japan, Ishigaki, Okinawa 907-0024, Japan \\ ${ }^{4}$ Nishi-Harima Astronomical Observatory, Center for Astronomy, University of Hyogo, Sayo, Hyogo 679-5313, Japan \\ ${ }^{5}$ Institute of Space and Astronautical Science (ISAS), Japan Aerospace Exploration Agency (JAXA), Sagamihara, Kanagawa 252-5210, Japan \\ ${ }^{6}$ Department of Cosmosciences, Graduate School of Science, Hokkaido University, Sapporo 060-0810, Japan \\ ${ }^{7}$ Hiroshima Astrophysical Science Center, Hiroshima University, Higashihiroshima, Hiroshima 739-8526, Japan \\ ${ }^{8}$ National Astronomical Observatory of Japan, National Institute of Natural Sciences, Mitaka, Tokyo 181-8588, Japan \\ ${ }^{9}$ Faculty of Education, Saitama University, Sakura, Saitama 338-8570, Japan \\ ${ }^{10}$ Department of Physics, Tokyo Institute of Technology, Meguro-ku, Tokyo 152-8551, Japan \\ ${ }^{11}$ Institute of Astronomy, Graduate School of Science, The University of Tokyo, Mitaka, Tokyo 181-0015, Japan \\ ${ }^{12}$ Department of Astrophysics, Nagoya University, Chikusa-ku, Nagoya 464-8602, Japan \\ ${ }^{13}$ Department of Astronomy, Graduate School of Science, Kyoto University, Kyoto 606-8502, Japan \\ ${ }^{14}$ Graduate School of Science and Engineering, Kagoshima University, Kagoshima 890-0065, Japan \\ ${ }^{15}$ Hiroshima Astrophysical Science Center, Hiroshima University, 1-3-1 Kagamiyama, Higashi-Hiroshima, Hiroshima 739-8526, Japan \\ ${ }^{16}$ Department of Astronomy, Kyoto University, Kyoto 606-8502, Japan \\ ${ }^{17}$ National Astronomical Observatory of Japan, Mitaka, Tokyo 181-8588, Japan \\ ${ }^{18}$ Institut d'Astrophysique de l'Université de Liège, Allée du 6 Août 17, B-4000 Liège, Belgium \\ ${ }^{19}$ Observatoire de Paris, I.M.C.C.E., Denfert Rochereau, Bat. A., F-75014 Paris, France \\ Received 2014 October 12; accepted 2014 December 11; published 2014 December 31
}

\begin{abstract}
We report a new observation of the Jupiter family comet 209P/LINEAR during its 2014 return. The comet is recognized as a dust source of a new meteor shower, the May Camelopardalids. 209P/LINEAR was apparently inactive at a heliocentric distance $r_{h}=1.6 \mathrm{AU}$ and showed weak activity at $r_{h} \leqslant 1.4 \mathrm{AU}$. We found an active region of $<0.001 \%$ of the entire nuclear surface during the comet's dormant phase. An edge-on image suggests that particles up to $1 \mathrm{~cm}$ in size (with an uncertainty of factor 3-5) were ejected following a differential power-law size distribution with index $q=-3.25 \pm 0.10$. We derived a mass-loss rate of $2-10 \mathrm{~kg} \mathrm{~s}^{-1}$ during the active phase and a total mass of $\approx 5 \times 10^{7} \mathrm{~kg}$ during the 2014 return. The ejection terminal velocity of millimeter- to centimeter-sized particles was $1-4 \mathrm{~m} \mathrm{~s}^{-1}$, which is comparable to the escape velocity from the nucleus $\left(1.4 \mathrm{~m} \mathrm{~s}^{-1}\right)$. These results imply that such large meteoric particles marginally escaped from the highly dormant comet nucleus via the gas drag force only within a few months of the perihelion passage.
\end{abstract}

Key words: comets: individual (209P/LINEAR) - interplanetary medium

\section{INTRODUCTION}

The link between comets and meteor showers is important for better understanding of how pristine cometary materials have been delivered to the Earth. 209P/LINEAR (hereafter 209P) has an orbit typical of Jupiter family comets, that is, a semimajor axis $a=2.932 \mathrm{AU}$, eccentricity $e=0.692$, inclination $i=199^{\circ} .4$, and Tisserand parameter with respect to Jupiter, $T_{\mathrm{J}}$, of 2.80 . It was suggested that a swarm of dust from 209P might cause a meteor shower on UT 2014 May 24 (Jenniskens \& Lyytinen 2014). Ye \& Wiegert (2014) has reported that 209P is relatively depleted in dust production, with a low level of activity around the perihelion passage in 2008 and 2009. This Letter attempts to characterize the physical properties further through a new observation in 2014. We focus on the dust ejection properties (e.g., particle size and ejection terminal velocity), which are

\footnotetext{
${ }^{20}$ Visiting Astronomer, Observatoire de Paris, I.M.C.C.E., Denfert Rochereau,
} Bat. A., F-75014 Paris, France, in 2014 May-July. pivotal for linking the comet with the meteor shower via a dynamical model (see, e.g., Vaubaillon \& Colas 2005).

\section{OBSERVATIONS AND DATE ANALYSIS}

The journal of these observations is summarized in Table 1. The first imaging observation was conducted on UT 2014 February 1 using a Tektronix $2048 \times 2048$ pixel CCD camera (Tek2k) on the University of Hawaii $2.24 \mathrm{~m}$ telescope (UH2.2 m) atop Mauna Kea. We obtained optical images with a broadband Kron-Cousins $R_{\mathrm{C}}$-band filter. We noticed that the comet appeared point-like even at a heliocentric distance $r_{h}=1.57 \mathrm{AU}$, where comets generally display comae and tails. Later, we made a network observation through the Optical and Infrared Synergetic Telescopes for Education and Research (OISTER), which is an inter-university observation network in the optical and infrared wavelengths. Among the OISTER network, we used four telescopes for the present study: the Nishi-Harima Astronomical Observatory Nayuta $2.0 \mathrm{~m}$ telescope (NHAO $2 \mathrm{~m}$ ), the Ishigakijima Astronomical Observatory Murikabushi $1.0 \mathrm{~m}$ 
Table 1

Observation Summary

\begin{tabular}{|c|c|c|c|c|c|c|c|c|c|c|}
\hline Median UT & Telescope & Filter & $N^{\mathrm{a}}$ & $T_{\text {tot }}^{\mathrm{b}}$ & $\mathrm{r}_{h}^{\mathrm{c}}$ & $\Delta^{\mathrm{d}}$ & $\alpha^{\mathrm{e}}$ & $f_{T}^{\mathrm{f}}$ & $\mathrm{Mag}^{\mathrm{g}}$ & Tail $^{\mathrm{h}}$ \\
\hline 2014 Feb 1.418 & UH $2.2 \mathrm{~m}$ & $R_{\mathrm{C}}$ & 72 & 216 & 1.572 & 0.729 & 27.6 & 272.7 & 17.6 & No \\
\hline 2014 Feb 16.540 & IAO $1.0 \mathrm{~m}$ & $g^{\prime}, R_{\mathrm{C}}, I_{\mathrm{C}}$ & 17 & 51 & 1.436 & 0.663 & 36.8 & 281.1 & 17.6 & No \\
\hline 2014 Feb 22.697 & IAO $1.0 \mathrm{~m}$ & $g^{\prime}, R_{\mathrm{C}}, I_{\mathrm{C}}$ & 17 & 51 & 1.381 & 0.641 & 40.8 & 285.0 & 17.4 & No \\
\hline 2014 Feb 28.598 & IAO $1.0 \mathrm{~m}$ & $g^{\prime}, R_{\mathrm{C}}, I_{\mathrm{C}}$ & 19 & 57 & 1.330 & 0.622 & 44.8 & 289.0 & 17.2 & No \\
\hline 2014 Mar 03.855 & NHAO $2.0 \mathrm{~m}$ & $R_{\mathrm{C}}$ & 20 & 10 & 1.302 & 0.611 & 47.0 & 291.4 & 17.1 & No \\
\hline 2014 Mar 07.641 & NHAO $2.0 \mathrm{~m}$ & $R_{\mathrm{C}}$ & 55 & 27.5 & 1.271 & 0.598 & 49.5 & 294.2 & 17.2 & No \\
\hline 2014 Mar 16.551 & IAO $1.0 \mathrm{~m}$ & $g^{\prime}, R_{\mathrm{C}}, I_{\mathrm{C}}$ & 11 & 33 & 1.199 & 0.565 & 55.5 & 301.6 & 17.2 & No \\
\hline 2014 Mar 22.604 & IAO $1.0 \mathrm{~m}$ & $g^{\prime}, R_{\mathrm{C}}, I_{\mathrm{C}}$ & 24 & 72 & 1.154 & 0.539 & 59.6 & 307.1 & $\ldots$ & Yes \\
\hline 2014 Mar 23.660 & NHAO $2.0 \mathrm{~m}$ & $R_{\mathrm{C}}$ & 45 & 90 & 1.146 & 0.534 & 60.4 & 308.1 & 17.0 & Yes \\
\hline 2014 Apr 1.502 & OAO $0.5 \mathrm{~m}$ & $g^{\prime}, R_{\mathrm{C}}, I_{\mathrm{C}}$ & 53 & 53 & 1.087 & 0.488 & 66.6 & 317.0 & 17.1 & Yes \\
\hline 2014 Apr 02.618 & NHAO $2.0 \mathrm{~m}$ & $R_{\mathrm{C}}$ & 14 & 28 & 1.080 & 0.481 & 67.4 & 318.2 & 16.9 & Yes \\
\hline 2014 Apr 04.562 & IAO $1.0 \mathrm{~m}$ & $g^{\prime}, R_{\mathrm{C}}, I_{\mathrm{C}}$ & 18 & 54 & 1.068 & 0.470 & 68.9 & 320.3 & 17.0 & Yes \\
\hline 2014 Apr 04.620 & NHAO $2.0 \mathrm{~m}$ & $R_{\mathrm{C}}$ & 20 & 40 & 1.068 & 0.470 & 68.9 & 320.4 & 17.1 & Yes \\
\hline 2014 Apr 06.643 & NHAO $2.0 \mathrm{~m}$ & $R_{\mathrm{C}}$ & 41 & 82 & 1.057 & 0.457 & 70.4 & 322.6 & 16.5 & Yes \\
\hline 2014 Apr 09.514 & OAO $0.5 \mathrm{~m}$ & $g^{\prime}, R_{\mathrm{C}}, I_{\mathrm{C}}$ & 55 & 55 & 1.042 & 0.439 & 72.6 & 325.9 & 17.1 & Yes \\
\hline 2014 Apr 10.596 & IAO $1.0 \mathrm{~m}$ & $g^{\prime}, R_{\mathrm{C}}, I_{\mathrm{C}}$ & 20 & 60 & 1.036 & 0.432 & 73.4 & 327.2 & 16.8 & Yes \\
\hline 2014 Apr 16.534 & NHAO $2.0 \mathrm{~m}$ & $R_{\mathrm{C}}$ & 20 & 40 & 1.010 & 0.390 & 78.0 & 334.3 & 16.5 & Yes \\
\hline 2014 Apr 17.610 & IAO $1.0 \mathrm{~m}$ & $g^{\prime}, R_{\mathrm{C}}, I_{\mathrm{C}}$ & 18 & 54 & 1.006 & 0.382 & 78.8 & 335.6 & 16.6 & Yes \\
\hline 2014 Apr 18.547 & NHAO $2.0 \mathrm{~m}$ & $R_{\mathrm{C}}$ & 30 & 60 & 1.002 & 0.375 & 79.5 & 336.8 & 16.5 & Yes \\
\hline 2014 Apr 19.638 & NHAO $2.0 \mathrm{~m}$ & $R_{\mathrm{C}}, I_{\mathrm{C}}$ & 30 & 60 & 0.998 & 0.366 & 80.4 & 338.2 & 16.6 & Yes \\
\hline 2014 Apr 22.549 & NHAO $2.0 \mathrm{~m}$ & $R_{\mathrm{C}}$ & 32 & 63 & 0.989 & 0.343 & 82.7 & 341.9 & 16.2 & Yes \\
\hline 2014 Apr 24.503 & OAO $0.5 \mathrm{~m}$ & $g^{\prime}, R_{\mathrm{C}}, I_{\mathrm{C}}$ & 36 & 72 & 0.984 & 0.328 & 84.3 & 344.4 & 16.8 & Yes \\
\hline 2014 Apr 25.544 & NHAO $2.0 \mathrm{~m}$ & $R_{\mathrm{C}}$ & 40 & 40 & 0.982 & 0.319 & 85.1 & 345.7 & 16.4 & Yes \\
\hline 2014 Apr 29.572 & IAO $1.0 \mathrm{~m}$ & $g^{\prime}, R_{\mathrm{C}}, I_{\mathrm{C}}$ & 11 & 33 & 0.974 & 0.286 & 88.3 & 351.0 & 16.2 & Yes \\
\hline 2014 May 02.486 & OAO $0.5 \mathrm{~m}$ & $g^{\prime}, R_{\mathrm{C}}, I_{\mathrm{C}}$ & 36 & 72 & 0.971 & 0.260 & 90.6 & 354.9 & 16.0 & Yes \\
\hline 2014 May 03.584 & NHAO $2.0 \mathrm{~m}$ & $R_{\mathrm{C}}$ & 40 & 80 & 0.970 & 0.251 & 91.4 & 356.3 & 16.1 & Yes \\
\hline 2014 May 04.610 & NO $1.6 \mathrm{~m}$ & $R_{\mathrm{C}}$ & 11 & 17 & 0.970 & 0.242 & 92.2 & 357.7 & 15.8 & Yes \\
\hline 2014 May 10.491 & OAO $0.5 \mathrm{~m}$ & $g^{\prime}, R_{\mathrm{C}}, I_{\mathrm{C}}$ & 36 & 72 & 0.971 & 0.190 & 96.3 & 5.6 & 15.8 & Yes \\
\hline 2014 May 10.534 & NHAO $2.0 \mathrm{~m}$ & $R_{\mathrm{C}}$ & 27 & 40.5 & 0.971 & 0.190 & 96.3 & 5.6 & 15.6 & Yes \\
\hline 2014 May 15.531 & NHAO $2.0 \mathrm{~m}$ & $R_{\mathrm{C}}$ & 11 & 16.5 & 0.978 & 0.145 & 98.9 & 12.2 & 15.3 & Yes \\
\hline 2014 May 16.542 & NHAO $2.0 \mathrm{~m}$ & $R_{\mathrm{C}}$ & 40 & 60 & 0.980 & 0.136 & 99.2 & 13.5 & 15.6 & Yes \\
\hline 2014 May 17.486 & OAO $0.5 \mathrm{~m}$ & $g^{\prime}, R_{\mathrm{C}}, I_{\mathrm{C}}$ & 45 & 45 & 0.983 & 0.128 & 99.4 & 14.8 & 15.4 & Yes \\
\hline 2014 May 17.541 & NHAO $2.0 \mathrm{~m}$ & $R_{\mathrm{C}}$ & 39 & 58.5 & 0.983 & 0.127 & 99.5 & 14.9 & 15.3 & Yes \\
\hline 2014 May 19.888 & NO $1.6 \mathrm{~m}$ & $R_{\mathrm{C}}$ & 12 & 8 & 0.988 & 0.110 & 99.5 & 17.4 & 14.4 & Yes \\
\hline 2014 May 21.547 & $\mathrm{OAO} 0.5 \mathrm{~m}$ & $g^{\prime}, R_{\mathrm{C}}, I_{\mathrm{C}}$ & 193 & 96.5 & 0.994 & 0.094 & 98.8 & 20.0 & 14.6 & Yes \\
\hline 2014 May 22.508 & OAO $0.5 \mathrm{~m}$ & $g^{\prime}, R_{\mathrm{C}}, I_{\mathrm{C}}$ & 73 & 73 & 0.997 & 0.087 & 98.0 & 21.2 & 14.6 & Yes \\
\hline 2014 May 23.541 & OAO $0.5 \mathrm{~m}$ & $g^{\prime}, R_{\mathrm{C}}, I_{\mathrm{C}}$ & 167 & 167 & 1.000 & 0.079 & 96.8 & 22.5 & 14.2 & Yes \\
\hline 2014 May 24.491 & OAO $0.5 \mathrm{~m}$ & $g^{\prime}, R_{\mathrm{C}}, I_{\mathrm{C}}$ & 26 & 26 & 1.004 & 0.073 & 95.2 & 23.7 & 13.8 & Yes \\
\hline 2014 May 25.504 & OAO $0.5 \mathrm{~m}$ & $g^{\prime}, R_{\mathrm{C}}, I_{\mathrm{C}}$ & 51 & 51 & 1.007 & 0.067 & 92.9 & 25.0 & 13.7 & Yes \\
\hline 2014 May 25.586 & IAO $1.0 \mathrm{~m}$ & $g^{\prime}, R_{\mathrm{C}}, I_{\mathrm{C}}$ & 11 & 33 & 1.008 & 0.066 & 92.7 & 25.1 & 13.1 & Yes \\
\hline 2014 May 27.522 & OAO $0.5 \mathrm{~m}$ & $g^{\prime}, R_{\mathrm{C}}, I_{\mathrm{C}}$ & 230 & 115 & 1.070 & 0.058 & 85.9 & 27.3 & 13.2 & Yes \\
\hline 2014 May 27.535 & NHAO $2.0 \mathrm{~m}$ & $R_{\mathrm{C}}$ & 92 & 23.25 & 1.016 & 0.058 & 86.2 & 27.5 & 13.1 & Yes \\
\hline 2014 May 28.006 & TRAPPIST $0.6 \mathrm{~m}$ & $R_{\mathrm{C}}$ & 2 & 6 & 1.017 & 0.057 & 84.3 & 28.0 & 12.7 & Yes \\
\hline 2014 May 28.477 & OAO $0.5 \mathrm{~m}$ & $g^{\prime}, R_{\mathrm{C}}, I_{\mathrm{C}}$ & 18 & 9 & 1.020 & 0.056 & 82.2 & 28.6 & 13.4 & Yes \\
\hline 2014 May 29.497 & OAO $0.5 \mathrm{~m}$ & $g^{\prime}, R_{\mathrm{C}}, I_{\mathrm{C}}$ & 52 & 26 & 1.024 & 0.055 & 77.6 & 29.8 & 13.0 & Yes \\
\hline 2014 May 29.504 & IAO $1.0 \mathrm{~m}$ & $g^{\prime}, R_{\mathrm{C}}, I_{\mathrm{C}}$ & 46 & 23 & 1.024 & 0.056 & 77.6 & 29.8 & 13.0 & Yes \\
\hline 2014 May 29.513 & NHAO $2.0 \mathrm{~m}$ & $R_{\mathrm{C}}$ & 105 & 26.25 & 1.024 & 0.056 & 77.6 & 29.8 & 13.1 & Yes \\
\hline 2014 May 30.489 & OAO $0.5 \mathrm{~m}$ & $g^{\prime}, R_{\mathrm{C}}, I_{\mathrm{C}}$ & 102 & 51 & 1.029 & 0.057 & 73.3 & 31.0 & 12.7 & Yes \\
\hline 2014 May 31.478 & OAO $0.5 \mathrm{~m}$ & $g^{\prime}, R_{\mathrm{C}}, I_{\mathrm{C}}$ & 52 & 26 & 1.033 & 0.059 & 69.4 & 32.2 & 12.8 & Yes \\
\hline 2014 Jun 1.506 & IAO $1.0 \mathrm{~m}$ & $g^{\prime}, R_{\mathrm{C}}, I_{\mathrm{C}}$ & 7 & 3.5 & 1.038 & 0.064 & 65.9 & 33.4 & 12.1 & Yes \\
\hline 2014 Jun 10.028 & TRAPPIST $0.6 \mathrm{~m}$ & $R_{\mathrm{C}}$ & 1 & 1 & 1.086 & 0.127 & 53.2 & 42.9 & 13.2 & Yes \\
\hline 2014 Jun 16.990 & TRAPPIST $0.6 \mathrm{~m}$ & $R_{\mathrm{C}}$ & 5 & 5 & 1.132 & 0.191 & 48.6 & 50.0 & 14.7 & Yes \\
\hline 2014 Jun 24.028 & TRAPPIST $0.6 \mathrm{~m}$ & $R_{\mathrm{C}}$ & 6 & 6 & 1.183 & 0.258 & 44.8 & 56.6 & 15.3 & Yes \\
\hline
\end{tabular}

Notes.

a Number of exposures.

b Total exposure time (minutes).

${ }^{\mathrm{c}}$ Heliocentric distance (AU).

d Geocentric distance (AU).

e Solar phase angle (deg).

f True anomaly (deg).

${ }^{\mathrm{g}} R_{\mathrm{C}}$-band magnitudes.

${ }^{\mathrm{h}}$ Is a tail clearly observed? 


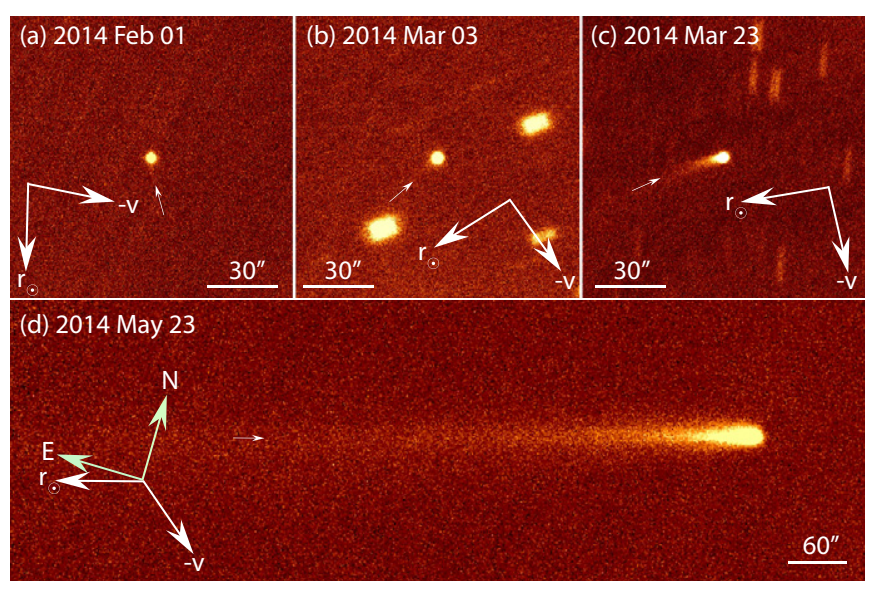

Figure 1. Selected images of 209P. The top three images (a)-(c) have the standard orientation in the sky: north is up, and east is to the left, and the bottom image (d) is rotated by $-17^{\circ}$ so that the Sun-comet vector is parallel to the horizontal axis. The FOV is $2^{\prime} \times 2^{\prime}(\mathrm{a}-\mathrm{c})$ and $144^{\prime} .5 \times 4^{\prime} .8(\mathrm{~d})$. The antisolar direction $\left(r_{\odot}\right)$ and the negative heliocentric velocity vector $(-v)$ are shown by arrows. Thin arrows indicate possible dust tails.

telescope (IAO $1 \mathrm{~m}$ ), the Okayama Astrophysical Observatory $0.5 \mathrm{~m}$ reflecting telescope $(\mathrm{OAO} 0.5 \mathrm{~m})$, and the Nayoro Observatory $1.6 \mathrm{~m}$ Pirka telescope of the Hokkaido University (NO $1.6 \mathrm{~m}$ ). We employed the optical imaging cameras MINT (a back-illuminated $2048 \times 2064$ CCD chip with a $15 \mu \mathrm{m}$ pixel pitch) with $R_{\mathrm{C}^{-}}$and $I_{\mathrm{C}}$-band filters at NHAO $2 \mathrm{~m}$, two sets of MITSuME $\left(g^{\prime}, R_{\mathrm{C}}\right.$, and $I_{\mathrm{C}}$ band simultaneous imaging system, a $1024 \times 1024$ CCD chip with a $24.0 \mu \mathrm{m}$ pixel pitch) at IAO $1 \mathrm{~m}$ and OAO $0.5 \mathrm{~m}$, and the MSI (a visible multispectral imager with a $512 \times 512$ CCD chip with a $16.0 \mu \mathrm{m}$ pixel pitch (Watanabe et al. 2012) at NO $1.6 \mathrm{~m}$. The two sets of MITSuME at IAO $1 \mathrm{~m}$ and OAO $0.5 \mathrm{~m}$ were designed identically, and each houses three front-illuminated CCD cameras. After early 2014 June, 209P was unobservable from these observatories, which are located in the northern hemisphere. Instead, we observed the comet with the $0.6 \mathrm{~m}$ Transiting Planets and Planetesimals Small Telescope (TRAPPIST $0.6 \mathrm{~m}$ ) with a $2048 \times 2048$ backilluminated CCD chip with a $15 \mu \mathrm{m}$ pixel pitch (Jehin et al. 2011). It covers $22^{\prime} \times 22^{\prime}$ with a resolution of $1^{\prime \prime} .3$ pixel $^{-1}$ using $2 \times 2$ binning. All telescopes were operated in a nonsidereal tracking mode so that the comet was stationary in the observed frames.

The observed data were analyzed in the standard manner for optical and near-infrared imaging data. We constructed medianstacked frames using 209P frames or dome flat images to correct for the effect of the pixel-to-pixel sensitivity variations across the detectors as well as optical vignetting (what is called the flat field image). The photometric zero levels were determined using Landolt photometric standard stars (Landolt 1992) for UH88 and NO1.6 m data and field stars listed in the USNO-A2.0 catalog (Mothé-Diniz et al. 2003) for the others. The images observed during a single night were combined to confirm the existence of a dust coma and further investigate the surface brightness profile of the dust tail (see Section 3.4).

\section{RESULTS}

\subsection{Appearance}

We found no significant morphological differences between the $g^{\prime}-, R_{\mathrm{C}^{-}}$, and $I_{\mathrm{C}^{-}}$band images taken with MITSuME. The obtained color indices, $g^{\prime}-R_{\mathrm{C}}=0.8 \pm 0.3$ and
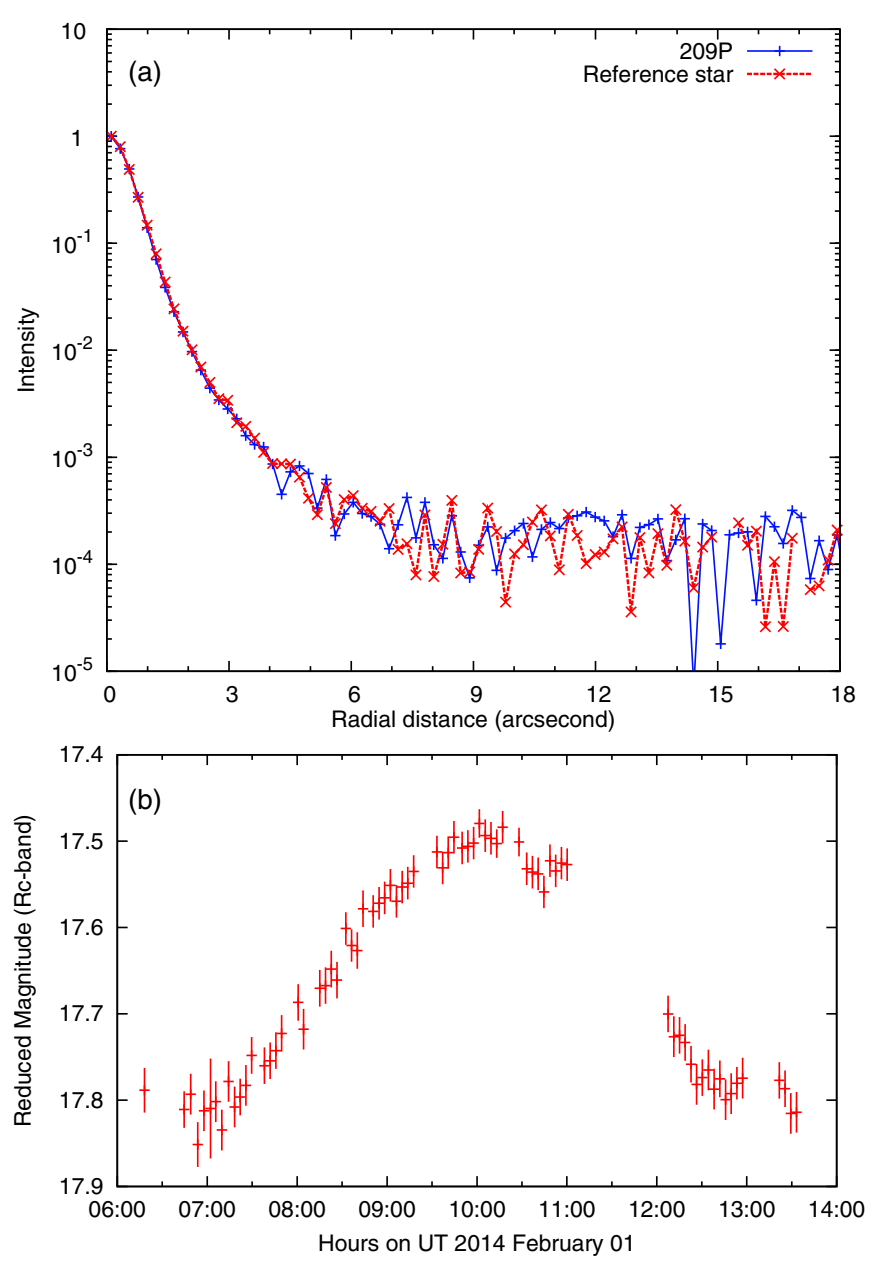

Figure 2. (a) Normalized surface brightness profiles of 209P (solid line) and a reference star (dashed line) taken on UT 2014 February 1. The stellar profile was taken in sidereal tracking mode six times at the beginning, middle and end of 209P exposures with the exposure time of $180 \mathrm{~s}$. We could not find a noticeable time-variation in the stellar profiles. (b) Rotational light curve on the same night. Vertical axis denote the reduced $R_{\mathrm{C}}$ magnitude, and horizontal axis denotes UT on 2014 February 1 after light time correction.

$R_{\mathrm{C}}-I_{\mathrm{C}}=0.5 \pm 0.3$, are consistent with those of the Sun, that is, $\left(g^{\prime}-R_{\mathrm{C}}\right)_{\odot}=0.65$ (Kim et al. 2012) and $\left(R_{\mathrm{C}}-I_{\mathrm{C}}\right)_{\odot}=0.33$ (Holmberg et al. 2006), which implies that the reflected light from the nucleus and dust are the dominant light sources of the detected intensity. In addition, it is reported that the spectrum taken with the $8 \mathrm{~m}$ Gemini North telescope on April 9.25 UT did not reveal obvious emission lines attributable to sources such as $\mathrm{C}_{2}$ around 4500-5600 $\AA$ and $\mathrm{NH}_{2}$ around 4900-6300 (Schleicher 2014). For these reasons, we ignored the contribution of gaseous emission in our $R_{\mathrm{C}}$-band data and used the $R_{\mathrm{C}}$-band magnitudes for the subsequent photometric analysis (see also Table 1).

Figure 1 shows selected $R_{\mathrm{C}}$-band images of 209P. In the first image, taken on UT 2014 February 1 (at $r_{h}=1.57 \mathrm{AU}$ ), neither the coma nor the dust tail was visually apparent. An unclear tail-like feature extended to the position angle (the angle on the celestial plane measured from north through east) P.A. $\sim 185^{\circ}$. It is not clear whether the feature was attributable to the cometary tail or an artifact such as a diffraction spike from the support vanes of the secondary mirror. The 1".05-1".06 FWHM of the field stars is in perfect agreement with the value of 1".05 in the combined 209P image. In Figure 2(a), we compare the radial profile of 209P in a composite image with that of a field star 
taken in sidereal tracking mode between the 209P exposures. We found that the surface brightness profiles coincided with one another at the $10^{-3}-10^{-2} \%$ level of the photocenter. The similarity suggests that the comet was highly dormant on that night (UT 2014 February 1). We set an upper limit of 0.01 for the parameter $\eta$, which is defined as the ratio of the coma cross section to the nucleus cross section. Adopting a model in Luu \& Jewitt (1992) and assuming the ejection of small dust particles (a radius of $a_{\mathrm{d}}=0.5 \mu \mathrm{m}$ ) that are embedded in surface water ice, we obtained approximate estimates for the dust production rate $M_{\mathrm{d}} \lesssim 0.01 \mathrm{~kg} \mathrm{~s}^{-1}$ and the fractional active area $f \lesssim 1 \times 10^{-5}$ on UT 2014 February 1 (see also Ishiguro et al. 2011), The obtained $f$ value is significantly lower than those of the typical Jupiter family comets ( $f>10^{-3}$; Tancredi et al. 2006).

In Figure 1(b) (UT 2013 March 03 at $r_{h}=1.30 \mathrm{AU}$ ), the comet still appeared point-like. However, a careful investigation revealed a faint tail-like structure extending to P.A. $=128^{\circ} \pm 3^{\circ}$, which is close to the position angles of the Sun-comet radius vector $\left(\right.$ P.A. $\left.=123^{\circ}\right)$ but deviates slightly to the negative heliocentric velocity vector $\left(\right.$ P.A. $=216^{\circ}$ ). Since cometary dust tails usually appear between these two vectors, and the position angle does not align with the diffraction spike caused by the secondary mirror, we suspect that the extended structure might be a real cometary tail. In Figure 1(c) (UT 2013 March 23), the cometary tail was clearly detected. It extended to P.A. $=105^{\circ} \pm 4^{\circ}$, existing between the antisolar direction (P.A. $=100^{\circ}$ ) and the negative heliocentric velocity vector $\left(\right.$ P.A. $\left.=193^{\circ}\right)$. We detected an obvious tail in all the images after UT 2013 March 22. Figure 1(d) was taken when the comet was viewed edge-on on UT 2013 May 23. Note that the image was rotated to align the projected orbital plane in the horizontal direction. The comet possessed a narrow tail extended to P.A. $=108^{\circ} \pm 1^{\circ}$, which coincided with the position angle of the orbital plane projected on the sky (P.A. = 107.4). The tail extended out of the field of view (FOV; i.e., $\left.>13^{\prime \prime}\right)$. Further, the dust cloud extended sunward by $30^{\prime \prime}$ (rightward in the image), probably because of the ejection of fresh dust particles toward the Sun. To obtain a crude estimate of the ejection velocity, we employed the formula $l=v_{e j}^{2} /\left(2 \beta g_{\odot}\right)$, where $l$ is the apparent length of the sunward tail, $v_{e j}$ is the terminal escape velocity of dust particles, $\beta$ is the ratio of the solar radiation pressure to the solar gravity, and $g_{\odot}$ is the solar gravity at the position of the comet (Jewitt \& Meech 1987). We obtained $1.1 \mathrm{~m} \mathrm{~s}^{-1}$ assuming $1-\mathrm{cm}$ particles and $3.4 \mathrm{~m} \mathrm{~s}^{-1}$ assuming $1 \mathrm{~mm}$ particles. The order of magnitude estimate for $v_{e j}$ is consistent with the result of another model described below (Section 3.4).

\subsection{Properties of Nucleus}

Figure 2(b) shows the light curves of 209P measured from each image on UT 2014 February 1. The data were calibrated using Landolt photometric standard stars, ensuring an absolute magnitude accuracy of 0.05 mag or less (Landolt 1992). The rotational light curve covered one peak and probably two troughs (both ends), suggesting that the rotational period is not shorter than the observational duration $(7 \mathrm{hr})$. The inferred rotational period is consistent with a report by Hergenrother in which he derived two alternative solutions of $10.930 \pm 0.015$ and $21.86 \pm 0.04 \mathrm{hr}$ (Green 2014). We calculated the corresponding amplitude at $\alpha=0^{\circ}$ using an empirical function (Zappala et al. 1990),

$$
A(0)=\frac{A(\alpha)}{1+m \alpha},
$$

where $A\left(0^{\circ}\right)$ and $A(\alpha)$ are the amplitudes at phase angles (Sun-comet-observer angles) of $0^{\circ}$ and $\alpha$, respectively, and $m$ is a correction coefficient for the amplitude, which has different values for S-, C-, and M-type asteroids. We adopted $m=0.015$, the value for $\mathrm{C}$-type asteroids, because the comet nucleus may have optical properties similar to those of C-type asteroids rather than S- or M-type asteroids. Substituting $m=0.015$ and $\alpha=$ 27.6 , we obtained an axis ratio of 1:1.25.

The magnitude is related to the effective (or mean) radius of the nucleus, $r_{n}$, by

$$
p_{\mathrm{R}} \Phi(\alpha) r_{n}^{2}=2.25 \times 10^{22} r_{h}^{2} \Delta^{2} 10^{-0.4\left(m_{\mathrm{R}}-m_{\odot}\right)},
$$

where $p_{\mathrm{R}}$ is the geometric albedo in the $R_{\mathrm{C}}$ band; $\Phi(\alpha)$ is the phase function; $r_{h}$ and $\Delta$ are the heliocentric and geocentric distances, respectively, in $\mathrm{AU}$; and $m_{\odot}=-27.1$ is the apparent $R_{\mathrm{C}}$ magnitude of the Sun. $\Phi(\alpha)$ is often assumed to be $\Phi(\alpha)=10^{-0.4 b \alpha}$, where $b$ is a parameter characterizing the phase slope (Belskaya \& Shevchenko 2000). We assumed $b=0.04 \mathrm{mag} \mathrm{deg}^{-1}$ and $p_{\mathrm{R}}=0.05$, and obtained the $R_{\mathrm{C}}$-band absolute magnitude $H_{\mathrm{R}}=16.24$ and $r_{n}=1.4 \mathrm{~km}$, or the dimension of $2.5 \times 3.2 \mathrm{~km}$. Although there are uncertainties in $b$ (from 0.035 to $0.045 \mathrm{mag} \mathrm{deg}^{-1}$; Belskaya \& Shevchenko 2000) and $p_{\mathrm{R}}$ (from 0.03 to 0.07; Kim et al. 2014), which cause a $40 \%$ error $(\sim 1 \mathrm{~km})$ in the size, the derived size is in good agreement with that determined by a radar observation, ${ }^{21}$ which reported dimensions of $2.4 \times 3.0 \mathrm{~km}$. The similarity may suggest that the comet was inactive on 2014 February 1 and has optical properties typical of comet nuclei. For comparison, We fit our data at low phase angle $(\alpha<40.8)$ using the $H-G$ formalism (Lumme et al. 1984; Bowell et al. 1989) when the comet was apparently inactive, and obtained $H_{\mathrm{R}}=16.11 \pm 0.26$ and $G=0.15 \pm 0.17$.

\subsection{Coma Photometry}

Figure 3(a) shows the $R_{\mathrm{C}}$-band reduced magnitude (a hypothetical magnitude observed at $1 \mathrm{AU}$ from both the Earth and the Sun) with respect to the phase angle. We set the aperture size for photometry to three times the FWHM of point sources $\left(5^{\prime \prime}-9^{\prime \prime}\right.$, depending on the sky conditions). In the figure, we considered the uncertainty of the magnitude on the basis of two factors: one is associated with the uncertainty of the magnitudes of comparison stars (0.25 mag for USNO-A2.0), and the other results from the rotation of the nucleus (a half amplitude of the light curve, $0.18 \mathrm{mag}$ ), because most of our data could not cover an adequate rotational phase (except the data from $\mathrm{UH} 2.2 \mathrm{~m}$ and NO1.6 m). The data taken on UT 2014 February $1(\alpha=27.6)$ have the smallest error not only because they were calibrated with appropriate standard stars in the Landolt catalog, but also because the data covered a substantial rotational phase for deriving the mean magnitude. In Figure 3(a), we show the reference magnitude of the nucleus, which is given by $m_{R}(\alpha)=16.24+0.04 \alpha$, following the result in Section 3.2. The magnitude of 209P was significantly brighter than the predicted nuclear magnitude at $\alpha \gtrsim 50^{\circ}$. Since we considered the rotational brightening/ darkening in the error bars, it is unlikely that the magnitude enhancement was caused by sampling bias. When we force fitted the magnitude data with a linear function, we obtained a phase slope of $b=0.03$, which is inconsistent with low-albedo objects (see Belskaya \& Shevchenko 2000). Therefore, it is reasonable to think that the magnitude enhancement was caused by a dusty coma near the nucleus.

\footnotetext{
21 http://www.usra.edu/news/pr/2014/comet209PLINEAR/
} 

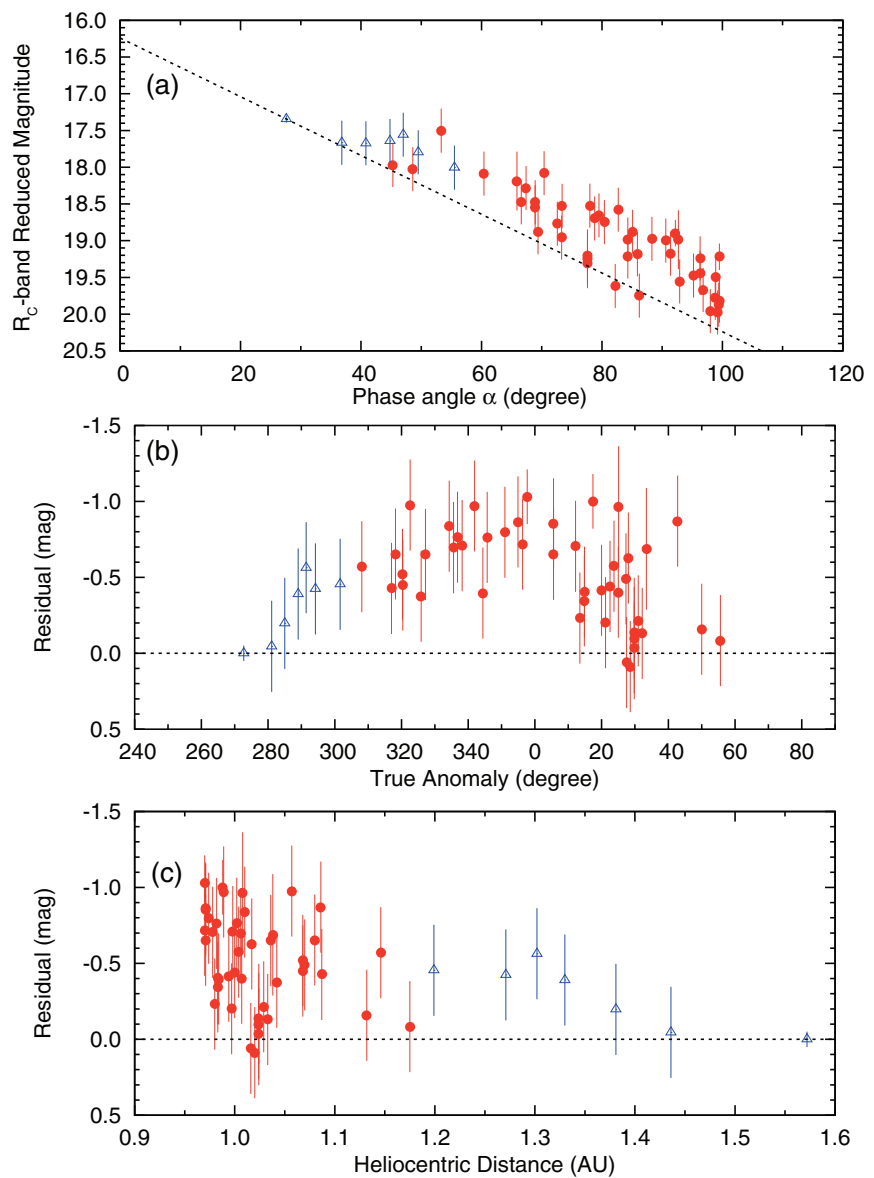

Figure 3. Photometric results: (a) Magnitude-phase relation of 209P/LINEAR. Dashed line denotes the predicted mean magnitude of the rotating nucleus. (b) Residual of magnitudes after subtraction of the nuclear contribution with respect to the true anomaly $\theta_{T}$. (c) Residual of magnitudes with respect to the distance from the Sun. Filled circles are magnitudes when the comet showed obvious tail while open triangles are magnitude when the comet appeared point-like.

Figures 3(b) and (c) show the differences in magnitude between the observation and the nucleus model with respect to true anomaly $\theta_{T}$ and the heliocentric distance $r_{h}$, respectively. There seems to be a weak trend that the residual increased toward perihelion (see Figure 3(c)). The magnitude enhancement appears at $r_{h}=1.2-1.4 \mathrm{AU}\left(\right.$ or $\theta_{T}=285-300^{\circ}$ ), although the tail was not obvious in our composite images. We conjecture that the nuclear magnitude was brightened at $r_{h}=1.2-1.4 \mathrm{AU}$ because of a thin dusty coma, although it was not noticeable in our images. It is thus likely that the tail-like feature in Figure 1(b) could be a dust tail associated with weak comet-like activity (see Section 3.1). We also noticed that the comet's activity may not be symmetric with respect to perihelion. The differential magnitude has a peak at $\theta_{T} \sim 340^{\circ}$, which is close to perihelion but slightly shifted toward the inbound orbit. Generally, activity peaks of comets tend to shift toward the post-perihelion passages (see, e.g., Ferrín 2010). We conjecture that the activity peak prior to the perihelion may be associated with the seasonal variation of solar incident flux at a localized active region, as indicated for 9P/Tempel 1 (Schleicher 2007).

\subsection{Dust Tail and Meteoroid Ejection}

To link a comet with a meteor shower, it is important to know how meteoric particles were ejected from the nucleus.
We determine the size and ejection velocity using a simple but straightforward method shown below.

We noticed that the edge-on image provides a unique opportunity for deriving the size and ejection velocity. It was taken on UT 2014 May 23 (Figure 1(d)) in a nearly edge-on view; that is, the angle between the observer and the 209P orbital plane was $3^{\circ}$. Figure 4 shows the surface brightness $(\Sigma)$ profile of the dust tail integrated within a width of $3^{\prime}$ perpendicular to the projected orbit, as a function of the distance from the nucleus, $d$. The profile at $d \lesssim 6^{\prime \prime}$ was contaminated by light from the nucleus. Since the comet moved rapidly on the sky plan $\left(12^{\prime}\right.$ minutes $\left.^{-1}\right)$, it was elongated up to $8^{\prime \prime}$ by inadequate tracking of the telescope.

In Figure 4, we found that an inflection point exists at $d \sim 50^{\prime \prime}$. The surface brightness along the tail is consistent with $\Sigma \propto d^{\gamma}$, where $\gamma=-0.57 \pm 0.05$ at $d=10^{\prime \prime}-50^{\prime \prime}$ and $\gamma=-0.73 \pm 0.03$ at $d=50^{\prime \prime}-300^{\prime \prime}$. Because the difference in $\gamma$ is significant to the accuracy of our measurement, we attribute the discontinuity at $d \sim 50^{\prime \prime}$ to a discontinuous distribution of dust particles. When dust particles are ejected at a constant rate over a long interval, the resulting steady-state flow of dust particles yields a surface brightness distribution with $\gamma=-0.5$. The similarity in $\gamma$ values between the observed data at $d=10^{\prime \prime}-50^{\prime \prime}$ and a steady-state flow suggests that dust particles flowed steadily owing to solar radiation pressure near the nucleus $\left(d<50^{\prime \prime}\right)$. In contrast, the steeper slope beyond $d=50^{\prime \prime}$ may suggest that only smaller particles reach the region, as considered in Jewitt et al. (2014). Assuming that the dust particles were ejected after late March at a constant rate, $d<50^{\prime \prime}$ corresponds to $\beta>3 \times 10^{-5}$ or $a \lesssim 1 \mathrm{~cm}$ (a density of $\rho=1 \mathrm{~g} \mathrm{~cm}^{-3}$ is assumed), where $\beta$ is again the ratio of the solar radiation pressure acceleration to solar gravity. We adopted the continuous dust ejection model in Jewitt et al. (2014) and found that the dust particles have a differential power-law size distribution with index $q \sim 3.25 \pm 0.10$. The ejection velocity perpendicular to the orbital plane was $0.7 \mathrm{~m} \mathrm{~s}^{-1}$ for $1 \mathrm{~cm}$ grains. Assuming that dust particles were ejected symmetrically to the comet-Sun vector within a half opening angle of $30^{\circ}-60^{\circ}$, the net ejection velocity is estimated to be $0.8-1.4 \mathrm{~m} \mathrm{~s}^{-1}$. With the model, we also estimated the ejection velocity of $1 \mathrm{~mm}$ particles as $2.5-4.4 \mathrm{~m} \mathrm{~s}^{-1}$. The velocity is consistent with or slightly faster than the escape velocity $\left(1.4 \mathrm{~m} \mathrm{~s}^{-1}\right)$ from an $1850 \mathrm{~m}$ body with a nuclear mass density of $1 \mathrm{~g} \mathrm{~cm}^{-3}$. Assuming that the dust has the same optical properties as the nucleus, we derived a total dust grain mass of $(2-8) \times 10^{7} \mathrm{~kg}$. Assuming that the particles were ejected for three months, from late March until late May, we obtained an average mass loss rate around perihelion of $2-10 \mathrm{~kg} \mathrm{~s}^{-1}$. The model predicts a loss of $2 \times 10^{8}$ particles s$^{-1}$ for $>1 \mathrm{~mm}$ particles. There seems to be an uncertainty of 3-5 times in the particle size due to the uncertain onset time of the active phase (i.e., late February or late March) and mass density $\left(0.3-2 \mathrm{~g} \mathrm{~cm}^{-3}\right)$. The uncertainty is translated into an uncertainty of $3-5$ in the particle production rate. Considering all of the results above, we concluded that meteoric particles $(1-10 \mathrm{~mm})$ were marginally ejected from the highly dormant comet nucleus via gas outflow only when the comet was around perihelion.

The peak activity of the Camelopardalids occurred on UT 2014 May 24 as predicted. Brown (2014) reported that the shower signals were dominated by small particles of milligram mass and smaller (i.e., $\lesssim 1 \mathrm{~mm}$ ). Further research is needed to connect the observed mass ejection for 209P and meteor shower, taking account of dynamical evolution (Vaubaillon \& 

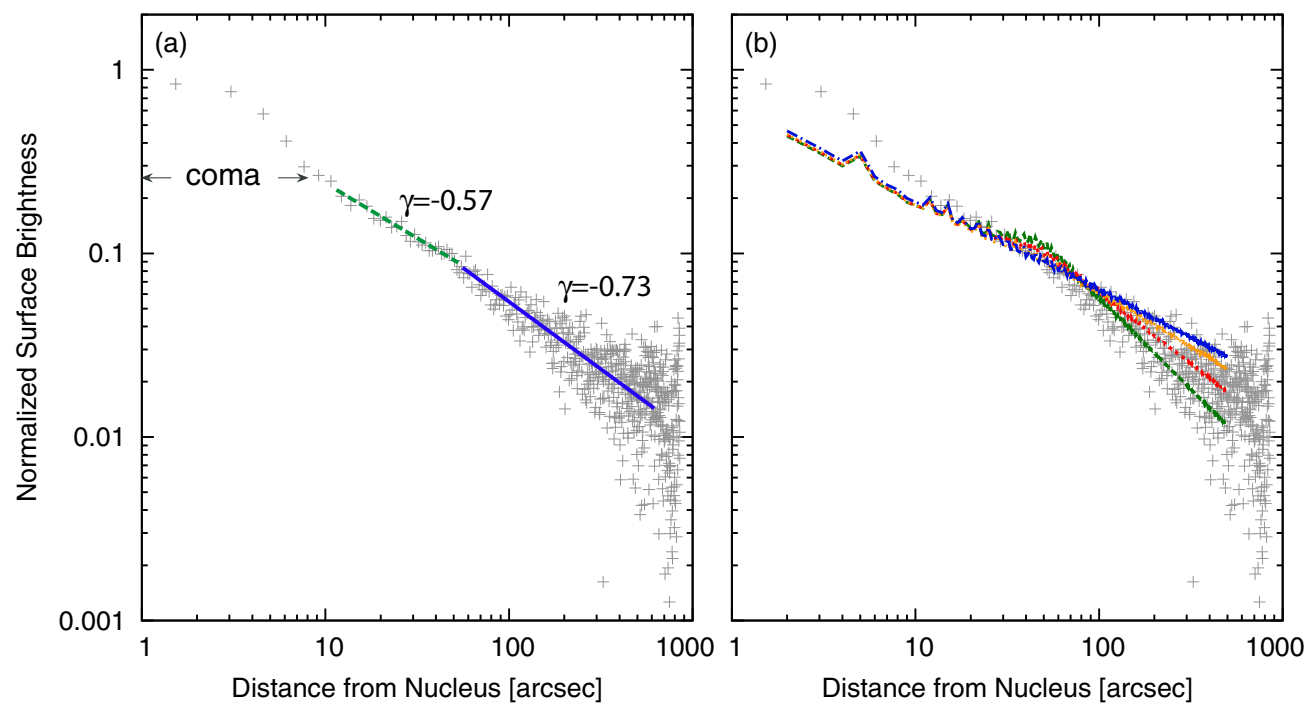

Figure 4. Surface brightness profiles of 209P (crosses) with respect to distance from the nucleus observed on UT 2014 May 23. (a) The profile was fitted by power-law functions with indexes $\gamma=-0.57\left(d=10^{\prime \prime}-50^{\prime \prime}\right)$ and $\gamma=-0.73 \pm 0.03\left(d=50^{\prime \prime}-300^{\prime \prime}\right)$. (b) Model profiles in which dust is ejected continuously starting on UT 2014 February 22. We assumed the minimum $\beta\left(=3 \times 10^{-5}\right)$, which corresponds to $1 \mathrm{~cm}$ grains, to produce the observed inflection point at $d \sim 50^{\prime \prime}$. The power-law indices are $q=-3.75$ (blue), -3.50 (orange), -3.25 (red), and -3.00 (green) from top to bottom.

Colas 2005) and probably fragmentation of dust aggregates (Madiedo et al. 2014).

\section{SUMMARY}

We made observations of 209P during its perihelion passage in 2014 and found the following.

1. 209P/LINEAR was apparently inactive at the heliocentric distance $r_{h}=1.6 \mathrm{AU}$ and showed weak activity at $r_{h} \leqslant$ $1.4 \mathrm{AU}$.

2. The observed morphology is similar in the $R_{\mathrm{C}}$ and $I_{\mathrm{C}}$ bands, suggesting that scattered sunlight from the nucleus and dust particles was the dominant light source at these optical wavelengths.

3. The comet was determined to have a negligibly small active fraction $(<0.001 \%)$ based on upper limit coma measurements made prior to the appearance of clear cometary activity.

4. During the active phase, it ejected dust particles up to $1 \mathrm{~cm}$ in size with a differential power-law size distribution with index $q=-3.25 \pm 0.10$.

5. The total ejected dust mass and average mass-loss rate were $(2-8) \times 10^{7} \mathrm{~kg}$ and $2-10 \mathrm{~kg} \mathrm{~s}^{-1}$, respectively.

This research was conducted as part of a joint research project titled "Recherche sur les liens entre comètes et météores." M.I. was supported by the Paris Observatory during his stay in Paris (2014 May-July). This research was also supported by a National Research Foundation of Korea (NRF) grant funded by the Korean government (MEST; No. 2012R1A4A1028713). The observations at OAO, IAO, NHAO, and NO were supported by the Optical and Near-infrared Astronomy Inter-University Cooperation Program and Grants-in-Aid for Scientific Research (23340048, 24000004, 24244014, and 24840031) from the Min- istry of Education, Culture, Sports, Science and Technology of Japan. TRAPPIST is a project funded by the Belgian Fund for Scientific Research (Fonds National de la Recherche Scientifique, F.R.S.-FNRS). C. Opitom acknowledges the support of the FNRS. E. Jehin and M. Gillon are FNRS Research Associates. S.H. was supported by the Space Plasma Laboratory, ISAS, JAXA.

\section{REFERENCES}

Belskaya, I. N., \& Shevchenko, V. G. 2000, Icar, 147, 94

Bowell, E., Hapke, B., Domingue, D., et al. 1989, Asteroids II (Tucson, AZ: Univ. Arizona Press), 524

Brown, P. 2014, CBET, 3886, 1

Ferrín, I. 2010, P\&SS, 58, 365

Green, D. W. E. 2014, CBET, 3881, 1

Holmberg, J., Flynn, C., \& Portinari, L. 2006, MNRAS, 367, 449

Ishiguro, M., Ham, J.-B., Tholen, D. J., et al. 2011, ApJ, 726, 101

Ivezić, Ž., Smith, J. A., Miknaitis, G., et al. 2007, AJ, 134, 973

Jehin, E., Gillon, M., Queloz, D., et al. 2011, Msngr, 145, 2

Jenniskens, P., \& Lyytinen, E. 2014, CBET, 3869, 1

Jewitt, D., Ishiguro, M., Weaver, H., et al. 2014, AJ, 147, 117

Jewitt, D. C., \& Meech, K. J. 1987, ApJ, 317, 992

Kim, J., Ishiguro, M., Hanayama, H., et al. 2012, ApJL, 746, L11

Kim, Y., Ishiguro, M., \& Usui, F. 2014, ApJ, 789, 151

Landolt, A. U. 1992, AJ, 104, 340

Lumme, K., Bowell, E., \& Harris, A. W. 1984, BAAS, 16, 684

Luu, J. X., \& Jewitt, D. C. 1992, Icar, 97, 276

Madiedo, J. M., Trigo-Rodríguez, J. M., Zamorano, J., et al. 2014, MNRAS, 445, 3309

Mothé-Diniz, T., Carvano, J. M. Á., \& Lazzaro, D. 2003, Icar, 162, 10

Schleicher, D. 2014, CBET, 3880, 1

Schleicher, D. G. 2007, Icar, 191, 322

Tancredi, G., Fernández, J. A., Rickman, H., \& Licandro, J. 2006, Icar, 182, 527 Vaubaillon, J., \& Colas, F. 2005, A\&A, 431, 1139

Watanabe, M., Takahashi, Y., Sato, M., et al. 2012, Proc. SPIE, 8446, 84462 O

Ye, Q., \& Wiegert, P. A. 2014, MNRAS, 437, 3283

Zappala, V., Cellino, A., Barucci, A. M., Fulchignoni, M., \& Lupishko, D. F. 1990, A\&A, 231, 548 